\title{
A Smart City Approach of Pedestrians' Spatial Experience in Night Markets using Space Syntax Analysis
}

\section{Camilo Ariel Jaime Gomez ${ }^{1}$}

National Taipei University of Technology, International Master Program of Interaction Design \& Innovation 1, Sec. 3, Zhongxiao E. Rd., Taipei 10608 Taiwan, R.O.C.

E-mail: t107at8409antut.edu.tw

\section{Sara Ines Sanchez Alquijay}

National Taipei University of Technology, Master Program of Interaction Design

1, Sec. 3, Zhongxiao E. Rd., Taipei 10608 Taiwan, R.O.C.

E-mail: t106ac8401entut. edu.tw

\section{Ryan Sheng-Ming Wang, Ph.D.}

National Taipei University of Technology, Associate Professor Department of Interaction Design

1, Sec. 3, Zhongxiao E. Rd., Taipei 10608 Taiwan, R.O.C.

E-mail: ryan5885email.ntut.edu.tw

Reported as one of the most visited top attractions in Taiwan, night markets are well-recognized for their diversity and very distinctive service model. After regular working hours have finished, these multiform commercial clusters emerge as an essential component of cities' urban fabrics. Many researchers have previously studied the walkable environment and physical activities in alternative study cases located in historical districts such as in the case of Kuala Lumpur, Malaysia (Mansouri \& Ujang, 2017), but very few have demonstrated the alteration in the space usage and the transformation suffered on street networks related to commercial zones after their reshaping caused by such informal business. This research has been conducted in a night market located in Taipei City, which is Taiwan's major urban centre and its most multicultural hub.

Understanding these unique commercial zones has appeared as a very important research topic within multiple disciplines. It also contributes to visualize vendors' business dynamics are directly affected by the perception that visitors visualize based on their experience. Therefore, learning about night markets' dynamics enhances (in different ways and professional fields) the improvement of the spatial experience regarding user-centered design and it promotes the venue as a worth visiting destination. This research initial results are certainly valuable contribution for a smart city approach with the integration of computational simulation and user-centered design.

Keywords:

Human-Centered Design; Smart City; Space Syntax; Spatial Experience; Night Market

International Symposium on Grids \& Clouds 2019, ISGC2019

31st March - 5th April, 2019

Academia Sinica, Taipei, Taiwan

${ }^{1}$ Speaker 


\section{Introduction}

Space syntax principle was introduced in 1976 by Hillier and his colleagues at University College of London (Hillier et al., 1976), the theory is based on a model where physical and spatial configuration of built environments report to us the way of how space is experienced, explored, apprehended and perceived. The mentioned outcome is achieved by analyzing a sequence of criteria and characteristics, space syntax assists designers to understand the role played by spatial notion in determining patterns of human behavior and therefore it estimates the social and mental effects on the proposed design alternatives.

This research first provides a theoretical and conceptual background that illustrates Taiwanese night markets' design logic and abstract urban sense. Having such information can provide an analysis of the reciprocity among visitors, vendors and the physical environment where they interact. There is enough funding to rely on the fact that having such analysis it is possible to innovate in the real-time suggestion of better routes and shopping experience in their intricate street networks. Enrichment in the application of space syntax gives increasingly exact visualization to the dispersion of passerby walking flow (Xiana, 2017).

Surveillance and site observation's data are intended to be supported and accompanied by the integration of space syntax analysis model (choropleth maps), this pursues to proof the way how computing technology provides valuable evidence to be integrated with smart city disciplines for further exploration, and subsequently its interpretation provides a tremendous impact on multiple aspects of service providers and customers' behavior.

\subsection{Research's Background and Motivation}

Based on the relevance of cultural entrepreneurship that night markets embody for Taiwanese culture. It's possible to highlight the fact that there are so many famous service offers with well-earned reputation all around the island, most of them come from long years operating tradition in the Taiwanese cuisine business. Such eateries are basically aligned along small aisles or dark allies, where sewage flows, these conditions directly affect the stores' quality. Thus, this initial study is motivated for categorizing and providing more options for pedestrian navigation through firstly, examining the spatial experience quality in the built environment where night markets operate. Secondly, identifying the mentioned quality in the pedestrians' accessibility to different business prospects, stalls and services offered in these venues.

Many must-visit spots in night markets are hidden or they just unfortunately don't offer their visitors a more comfortable condition for better customer's attraction. For newcomers or simply explorers the spatial arrangement and visibility of stalls and shops play a very decisive factor for their shopping or wandering route's selection. Designing and running this research also leads to the investigation to a wide range of different sort of factors like spatial configuration, attraction of pedestrians and their comfort which are cooperatively reflected with the traditional accessibility measures. 


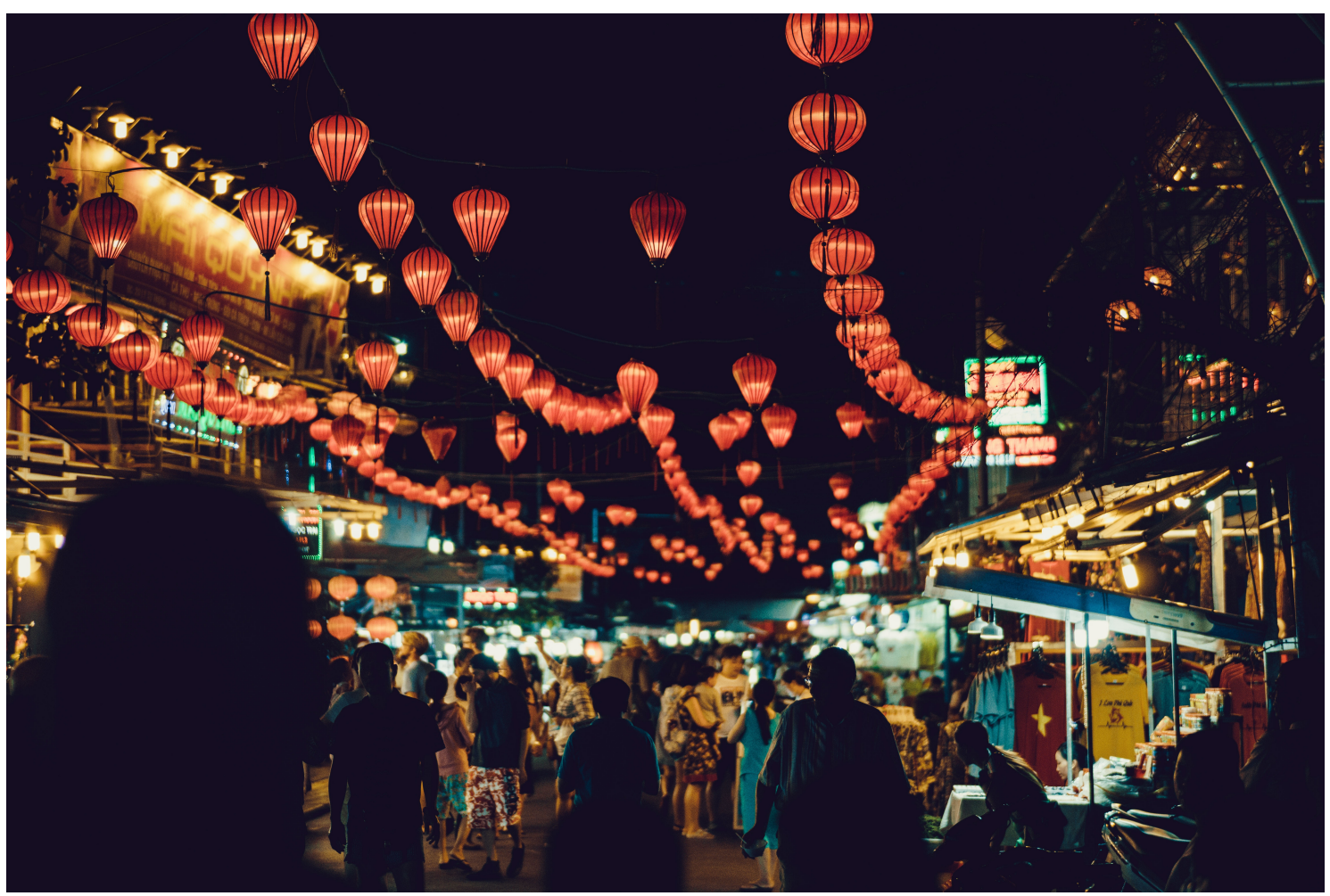

The smart city approach is an innovative way to provide a serviceable strategy to find the best routes or most accurate walking experience recommendation to wander through the street network in the area to reach the desired goal from the point of view of accessibility's quality. Balancing the walking visitors stream experience appropriately is the most accurate way for remodeling of business stores dynamics. As presented in (Era, 2012) concerning the mentioned scenario, the Space Syntax theory and other techniques developed at the Barlett School of the UCL introduce some measures and attributes. These elements could support the improvement in quality of the pedestrian experience of accessibility to the different destinations located within the built environment in night markets. The scope of the application of this research first results, comes as a proposal of merging the computing simulation outcome with an user-centered design theory to boost these destinations capacity to attract visitors. Therefore, in future it also represents an opportunity to suggest enhancement on the businesses' properties such as the stores' extension, design and service model facilities.

\subsection{Main Objectives}

As we know, today's night markets dynamic and moving forces are leading the operation of such particular retail businesses involved in this context. The visitor experience quality implies the need of a different approach from the traditional one that has been applied in previous accessibility researches. This research aims to the visualization of a wholesome merged version of integration value results as product of combining both global \& local values. For this research, it was strictly necessary to establish the objectives according the fields involved into consideration. Starting for the visitor's spatial experience attributes, secondly human-centered design, then in the "servicescape" domain to finally get into a smart city approach. 
According to Lynch (1960) accessibility is a time issue; but it also depends on the "attractiveness" and the identity of the itineraries. For the selected study case, accessibility is considered to be linked to the pedestrians. Thus, pedestrians movement is a mobility model that puts into practice a simulation showing where visitors have the capacity of engaging the highest level of interaction with the site's street network, e.g. through the five senses, in the interaction with other visitors, in the possibility of participating, in the trading activity and cultural undertaking one along this street networks (Ventury, 1998) and enjoying the natural, architectonic and spatial setup (Jacobs, 1996).

Therefore, it is seen the need of considering the pedestrian accessibility quality. There is a gap between accessibility and quality of the built environment, it might be translated into the context of a low integration between urban planning and mobility planning. From a business model point of view; street markets, bazaars and outdoor retailing generate positive externalities. Some authors, e.g. Correll, Lillydahl and Singell (1978); Luttik (2000); Kong et al (2007) highlight in their research how the areas near a public space with high quality of urban green space tend to get greater sale prices. For commercial zones in outdoors such as street markets in general, consumers' behavior doesn't simply arouse in retail spaces by default based on visual attraction properties but also are generally induced by corresponding space types (Hillier, 2007). This research pursues to prove that, under certain criteria when pedestrians' moving scheme is adjusted, the proper combination of integration value and vendor's distribution could anticipate the travelers' transit flow in a more successfully way.

Contrary to other studies where most of the analyses previously practised do not involve extensive data collection on actual densities of pedestrian movement (Özbil \& Peponis, 2007). The presented research suggests that the processed information obtained after practising the experiments can serve as an innovative way to support (slightly or considerably) different aspects of connectivity, accessibility and walkability pertinent to pedestrians' movement, and it also proportionally affects the street network and spatial variation caused by the mentioned phenomena throughout the studied area in the selected location.

\subsection{Project Importance}

According previous theories developed by the University College London - UCL the representations of layouts (as convex, axial or visibility graphs) and the topological and angular analysis seem to have a correspondence to the way humans cognize space, maybe because they reflect embodiment. In addition, shortened spatial association and reticular stalls, shops or ambulant carts' distribution promotes an adjusted stream transportation, space recognition has high importance when developing strategies for improving pedestrians' movement.

It is essential and necessary to establish that integration value is a measure for typological accessibility, during the simulations carried in this research this property correlates significantly with pedestrians' movement pattern and the isovist is considered as the volume of space visible from a given point in space, together with a specification of the location of that point, this property such as area consequently correlates with visitors' spatial behavior. 
Axial graphs were constructed for each of the night markets surroundings' scales, it was important to undestand the adjacent diferences between the macro and local ranges as well. This data - in effect a set of binary adjacency matrices - was taken as the computer's raw sense data, or experience of each of the spaces. The analysis was performed using depthmapX software, which constructed a minimal axial graph based entirely on plan input and an objective algorithm (Turner, Penn, Hillier, planning, \& design, 2005).

It pretends as well to contribute for a clear understanding of the help we can obtain from computation simulations to fight back against disasters, based on the simulated conditions to identify altered necessities of retail space management and adequate preparation to deal with urgent situations with all available resources to control pedestrian movement and flow within an infrastructure or a local neighborhood to avoid obstructions and accidents hazards.

\subsubsection{Pedestrians' Navigational Experience Behaviour}

Behavior can be described in terms of movement frequencies, distances between origins to destinations decline the movement frequency's observable properties of spatial acts (Golledge, 1997). It's conceivable to produce abundant descriptive data about the properties of distributions, network connections, patterns, interactions, nodes, surface properties and hierarchical elements of spatial systems.

Shopping and entertainment, both seen as the two main activities source of income in night markets are also changing. Following how technology progresses every day, computing automation \& convenient internet accessibility are stimulating shopping habits to evolve, consumer's spending behavior gradually becoming aligned with new developments, such developments play a very important role to bring data visualization forward to be interpreted creating a variety of utilization possibilities in near future.

Due to all the advances and developments we enjoy in the navigation field, users have full access to mobile applications and gadgets capable to provide real-time routes. People's spatial behavior when walking differs in many ways from any driver's routine (Millonig \& Schechtner, 2007), thus the usage of landmarks has vital importance for human navigation.

This mentioned situation is due to the fact that commonly navigating individuals often fail to find their way immediately under time pressure or other circumstances, such phenomenon brings up the importance of studying how to ensure the efficiency in the visitor's spatial experience, leading to individual benefits related to walking efficiency, environment safety and spending time reduction among others. Lastly, this research intends to call for further research to obtain an improved interpretation of pedestrian spatio-temporal behavior from a user-centered design perspective capable to be integrated with a smart city planning approach. 


\section{Literature Review}

Service design and branding patenting have clearly become a new tool for experience evaluation of businesses (Markopoulos, Read, MacFarlane, \& Hoysniemi, 2008). In any case, it is a late field that has emerged as merchandise with comparative usefulness started to enter into competition in the commercial arena. Choices made in merchandise configuration are regularly founded on viability, feasibility, and desirability. Certainly, the conception of attractive items has turned into an enormous worldwide business. As of late, the fields of service design and branding patenting has broadened its extension beyond physical items to address the conception of spatial encounters that can be appreciated through utilizing computer programming assistance. Computer-assisted simulations are thriving in the area of spatial morphological studies, same fields have strong relationship and contribution to this research.

It has been previously reported (Xiana, 2017), that making an exact forecast regarding to the commercial zone that individuals' flow allocation in the predefined spaces ensures accomplishing ideal business impacts of the trade condition. Therefore, it is conceivable to adequately foresee the appropriations of individuals' walking-streams (Hillier et al., 1993) to make sensible accommodations of spaces. Because the motion of individuals in night markets' streets network and paths are identified through the sense of emotions, their distinctive spatial allocation drives actions which give the trades' conditions probably not just the correct level of feasibility and spatial unit of various attractions. Yet, they also cause an incredible impact on the shopper's spreading in the adjacent roads and paths.

\subsection{Smart City and Space Syntax}

Smart cities' theory is proposed as a conceptual urban development model for the improvement of urban agglomerations. Unfortunately, the application of such principles still remains as a very abstract idea due to its lack of exploration within interdisciplinary fields. Specifically, in the case of pedestrian navigation for a smart city approach, there are studies that already have designed iterative mechanisms through adapting systematic techniques to give a wide range of solutions for creating the cities of tomorrow. In this research we intend to harmonize smart city with the computing simulation analysis carried out through space syntax.

Space syntax contributes into the perception of treating cities as spatial networks and also it tries to establish a relationship between the concept of space and the experiences involved into it. Thus, space is addressed as the common medium of both; the physical built environment city and the experiential city, as well as, of course the social economical urban conglomerate and the cognitive urban cluster. Then, spatially talking; the physical and experiential town merge into one. In fact, researchers have come up to the conclusion that cities are human products in a very deep insight. Particularly linked to human lifestyles in their very pure form and function, reflecting the way we satisfy our necessities what we do, how we solve our problems, becoming in an extended use of words: the greatest artifacts humanity gives to the world. 


\section{Methodology}

A space syntax approach provides another, different perspective of space. From a technical standpoint, it is basically a visualization of a graph-oriented representation of a geo-located point or area in space that models the free environments and possible pedestrians' flow within an urban system. Drawing an automatically generated axial map visualizing the "Fewest-Line Map" is a relatively straightforward task. The procedure starts by adding the polygon map drafted in AutoCAD or any other software of computer aid drawing to a new file completely clean from unwanted blocks or unnecessary elements, after having the geometric shapes, they are introduced to the DepthmapX computing for the analysis. This research puts into practice the implemented assistance of a computational anticipated representation to visualize the site visitors' circulation, such tool has great potential for scaleable applications in other cases as well, it has been utilized to analyze interior spaces and larger street networks.

The designed methodology for this research has four very well established phases: (1) data collection, (2) interpretation of the collected data, (3) association of the obtained computational information, (4) proposal of the gathered statements from the processing. The data collection phase was carried out from an observational and experimental perspective. Understanding the urban fabrics operational behavior is a rewarding process, the most prominent benefit has been the filtering of the night market candidates to finally choose the most advantageous for this research. Secondly, we have the interpretation of the global and local integration values obtained from introducing the roadways' fabric layout into the space syntax analysis. Such interpretation is product of the offered visualization graphics obtained after utilizing depthmapX software.

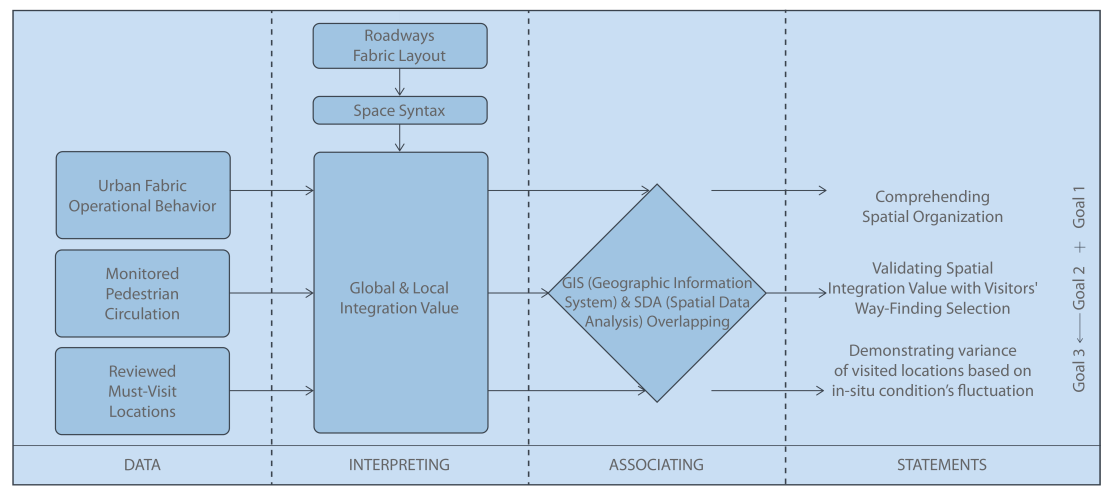

The third and fourth phases have a well established connection due to the association of geographic information system and the spatial data analysis overlapping to produce a clear visualization of the phenomenon simulated: the pedestrians' possible distribution throughout the selected area of the night market. In this specific phase, a correlation analysis was performed to outline the relationship between the obtained result of both integration value (connectivity aspect) of street networks in the system \& pedestrian circulation patterns. 
Finally, a comparison between number of visitors at each entrance in the studied zone was completed by in-situ observation to bring out their relationship. It was preliminary found out that there is a need to sensitively improve the organization of space in the night market to maximize pedestrians' exposure and experiences of these built environments.

\subsection{Research Methodological Framework}

In order to ensure the integration of the analyzed data and the research scope, it was decided to draw four major steps considering quantitative exploration tools, they were explained in the previous introduction. From the research's whole process, space syntax analysis played a very important role during the interpretation phase. With its assistance, visibility value of the final reduced axial map is analyzed, the analysis is helpful to further verify the rationality of the open space of the streets network, at the same time to determine flow of practical activities in the environment: from the visitor's favorable experience, high visibility provides high value to the business. Thus, it was necessary to work with two scales of integrity value.

Based on the presented scenario, the space syntax tool was conducted to obtain the axial model, and once the model has been reduced to the integration value grid it was permissive for the visualization of the different levels of accessibility in the street network.

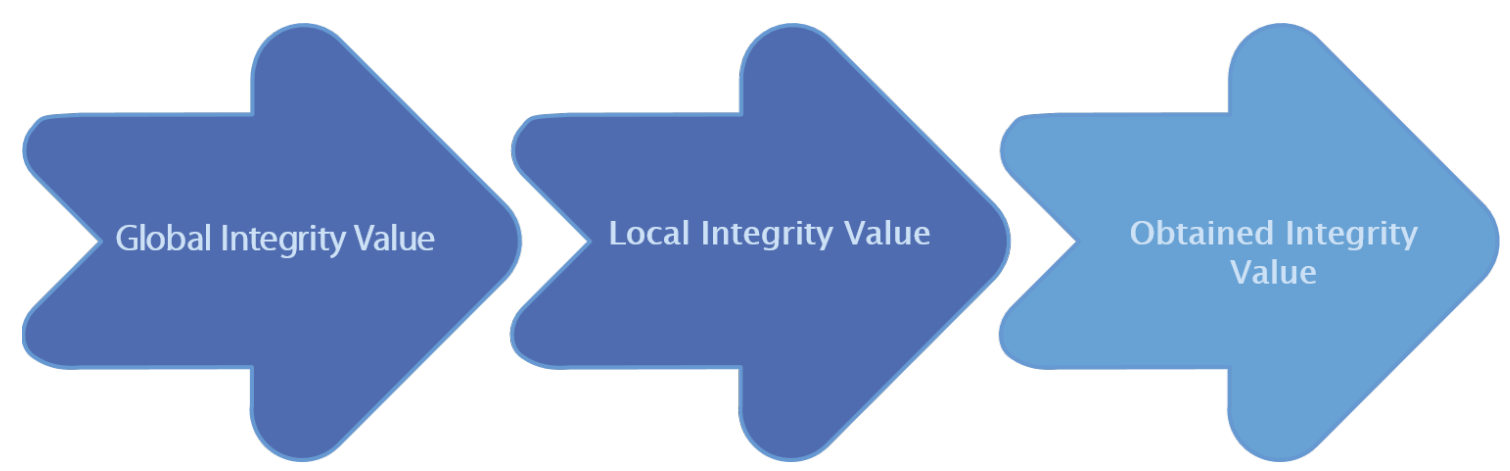

\subsection{In-Situ Observation and Number of Visitors' Calculation}

The first phase of data gathering was to survey the amount of visitors based on the doorcount method in one of the two official entrances of the night market, it was put into practice the discrimination out of the intended visitors' count without vendors among the people in the surroundings. The second phase involved the analysis of pedestrians' flow on streets network connectivity through the integration of global and local value analysis, both were adjusted to the different scales used to involve the research area using the space syntax assessment technique.

This part of the research specifically helped us to visualize visitors' movement patterns. This phases assisted us to create a visitors' movement database, such evidence collecting method is the same element directly contributing to the understanding of urban performance. The process started by data gathering to survey visitors' activities as pedestrians. The technique properly introduced by the UCL Space Syntax Laboratory, portal observation is a method of spatial observation. It consists of counting the pedestrians on a particular road in a specific time. 


\subsection{Research Method Workflow}

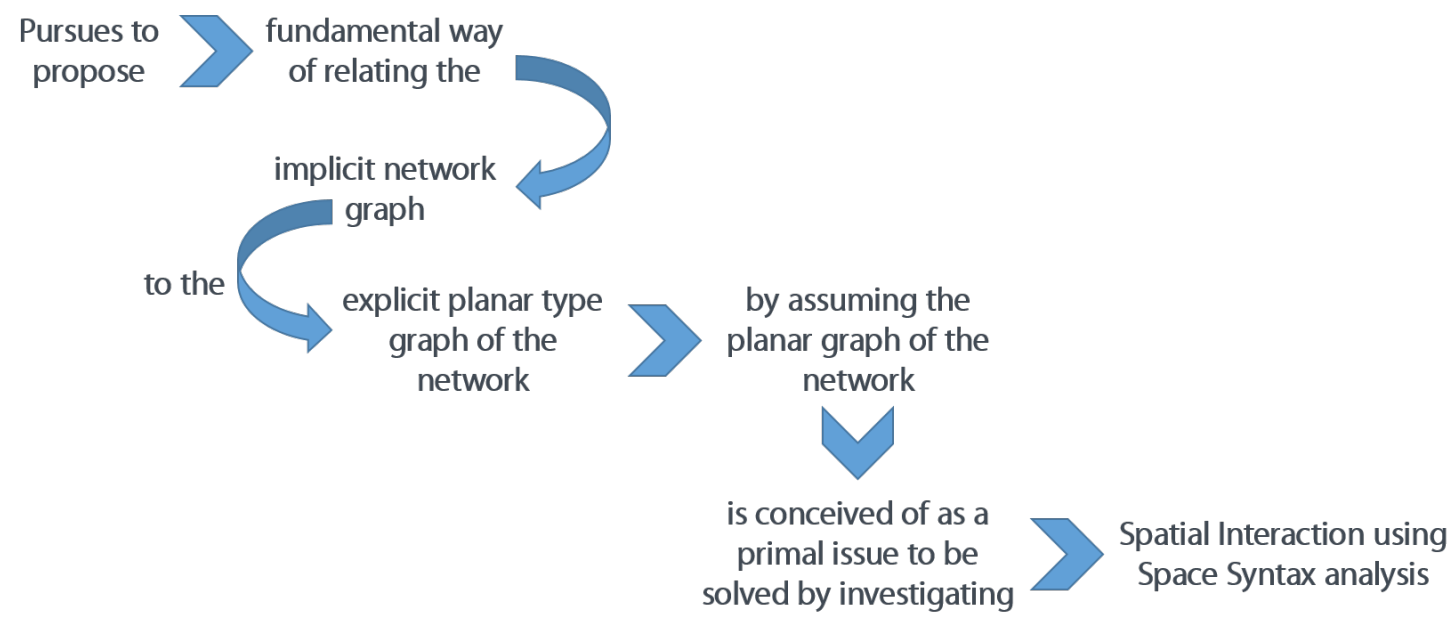

This research method workflow intends to propose a fundamental way to correlate the implicit network graph to the explicit planar type graph of the selected area's street network. It is planned to reach this proper work progress by assuming that the planar graph of the network involved is conceived of as a primal issue to be solved by investigating the spatial interaction happening within the night market's limits, this is tended to be carried out by the space syntax analysis. It is clear that walking is the main mode of experiencing the place, therefore accessibility and connectivity in night markets play a fundamental role in destination choice by visitors wandering throughout local facilities and attractions.

\subsection{Case Study}

The research work is developed on the street network of the Raohe Night Market in the Songshan District in Taipei City, Taiwan. The research's domain for the spatial scope and activities included into consideration are contained in a diverse expression of public spaces, e.g. squares, alleys, lanes \& boulevards. The night market is a $600 \mathrm{~m}$ path along Raohe street. It is packed with fun and interesting food and snack options, traditional shops and stalls, it also has plenty carnival games and prizes. Its locality has a strong connection to nearby transportation hubs of metro system, train station and city buses. In order to establish a clear link between the need of understanding the spatial variations through the proposed visualized approach with the quantitative properties of spatial experience, after reviewing previous study cases ( $\mathrm{Li}, \mathrm{Xiao}, \mathrm{Ye}$, $\mathrm{Xu}, \&$ Law, 2016), it is perceptible that the main method utilized in urban morphological and behavior research is space syntax.

\subsection{Implementation Platform: depthmapX}

This research particularly aims to illustrate the fluctuation between two different scenarios through the application of space syntax analysis: one according a regular daytime setting and the other during the night market's operating time, both by putting into practice two methods: visitor's surveillance and in-situ observation, where the mentioned fluctuation is subject to a conducted simulation by inputting certain criteria utilizing depthmapX software implementation. 
The selected implementation software is a multi-platform computational program capable to perform a set of spatial network analyses designed to understand social process within the circumscribed built environment. It works at a variety of scales from interior of buildings to whole cities, macro-regions or even states.

Big Scale Half-kilometer surroundings Fewest-Line Map (Minimum)

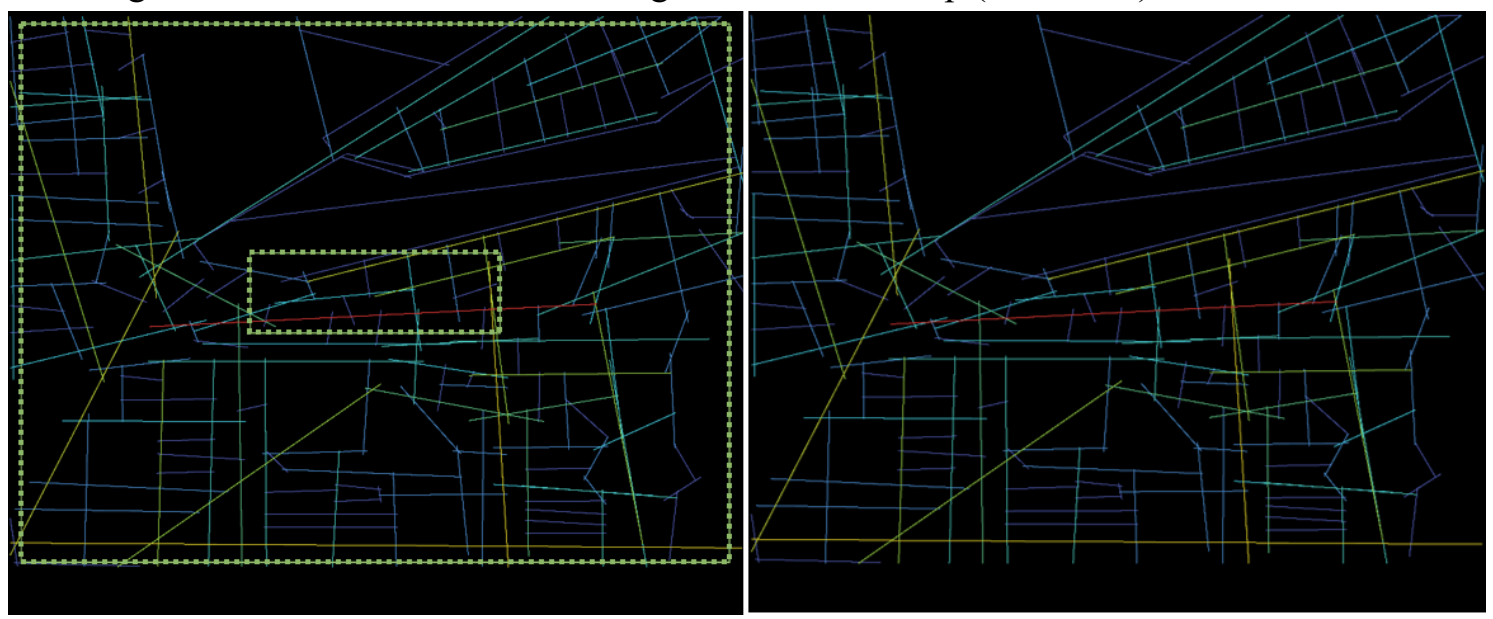

Local Scale (Night Market Area) Generated Axial Map
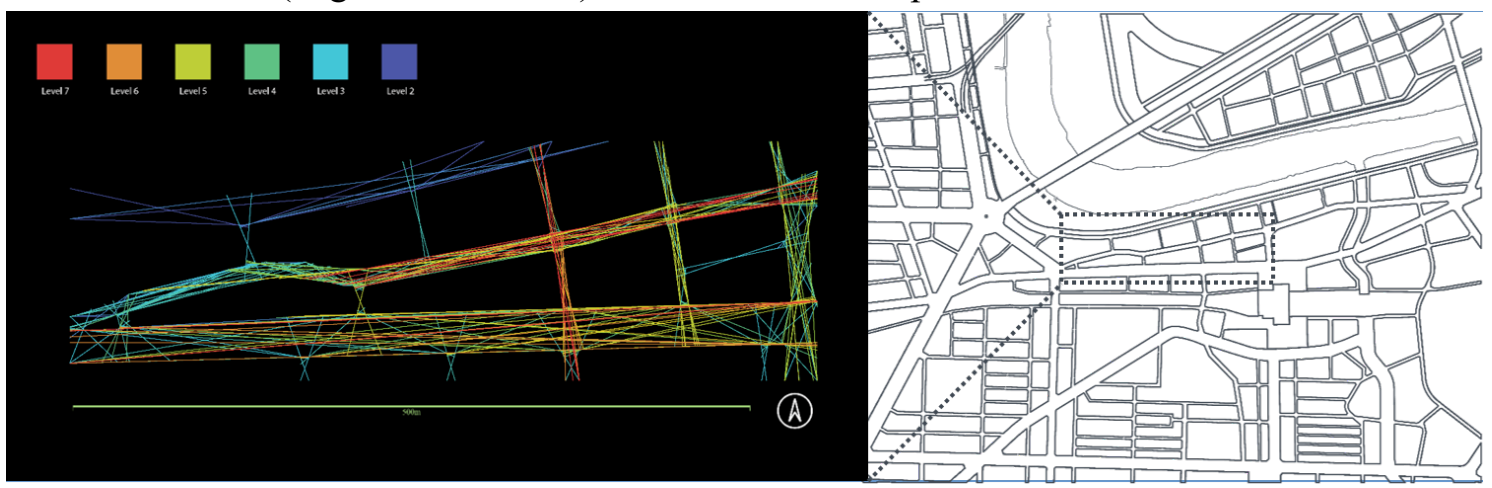

Local Scale (Research Area) Generated Axial Map Fewest-Line

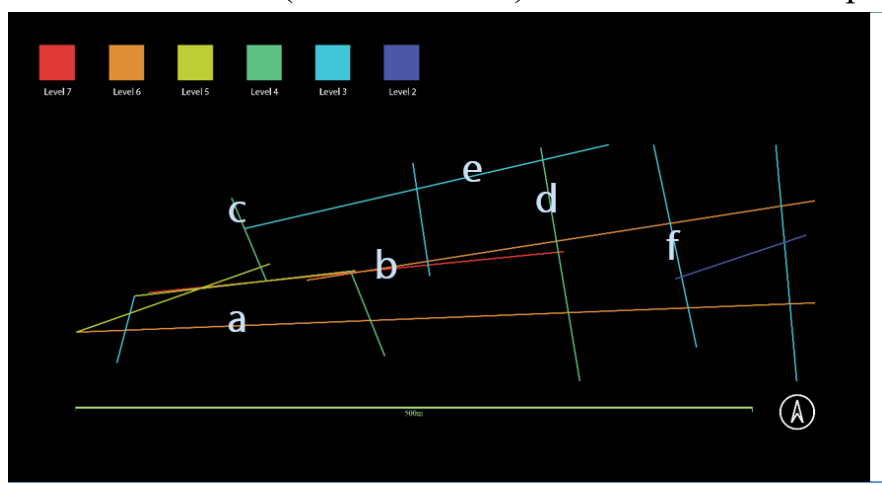

a. Section 4, Bade Road

b. Raohe Street

c. Lane 61, Raohe Street

d. Lane 221, Raohe Street

e. Songhe Street

f. Lane 709, Section 4, Bade Rd.

Visibility Graph Analysis (VGA)

This kind of analysis is the core of the space syntax theory. It is proposed to recast the adjacent matrix as a visibility graph of sites (points of interest, nodes, paths) where the grid edge connects vertices representing mutually visible locations. Then it takes in charge the responsibility of correlating them with movement patterns. Each graph provides the most accurate visualization of a computing layout from the selected space, and thus it expresses the real properties of the layout. 


\section{First Moment Visibility \& Second Moment Visibility}
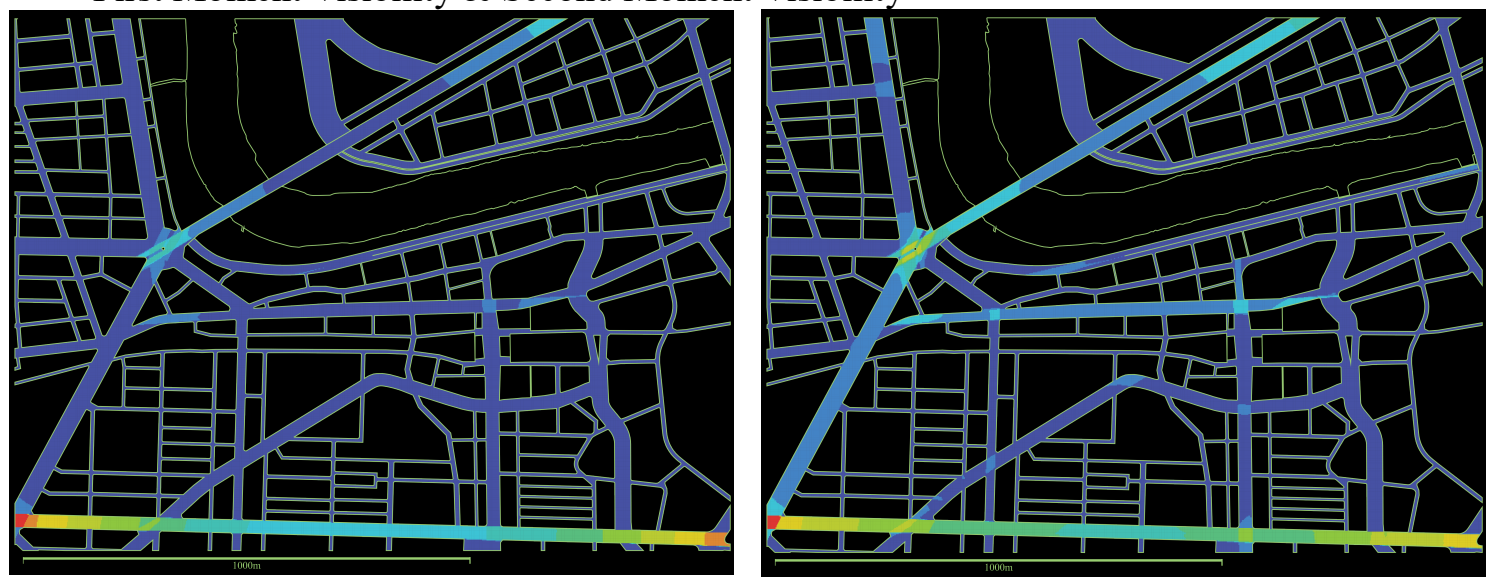

Third Moment Visibility

In VGA, a grid of points is overlaid on the plan. A graph is then made of the points, where each point is connected to every other point that it can see. The visual integration of a point is based on the number of visual steps it takes to get from that point to any other point within the system. In this analysis, 'agents' (automata within a computer representing people) are released into a plan of the environment and navigate using the visibility information.

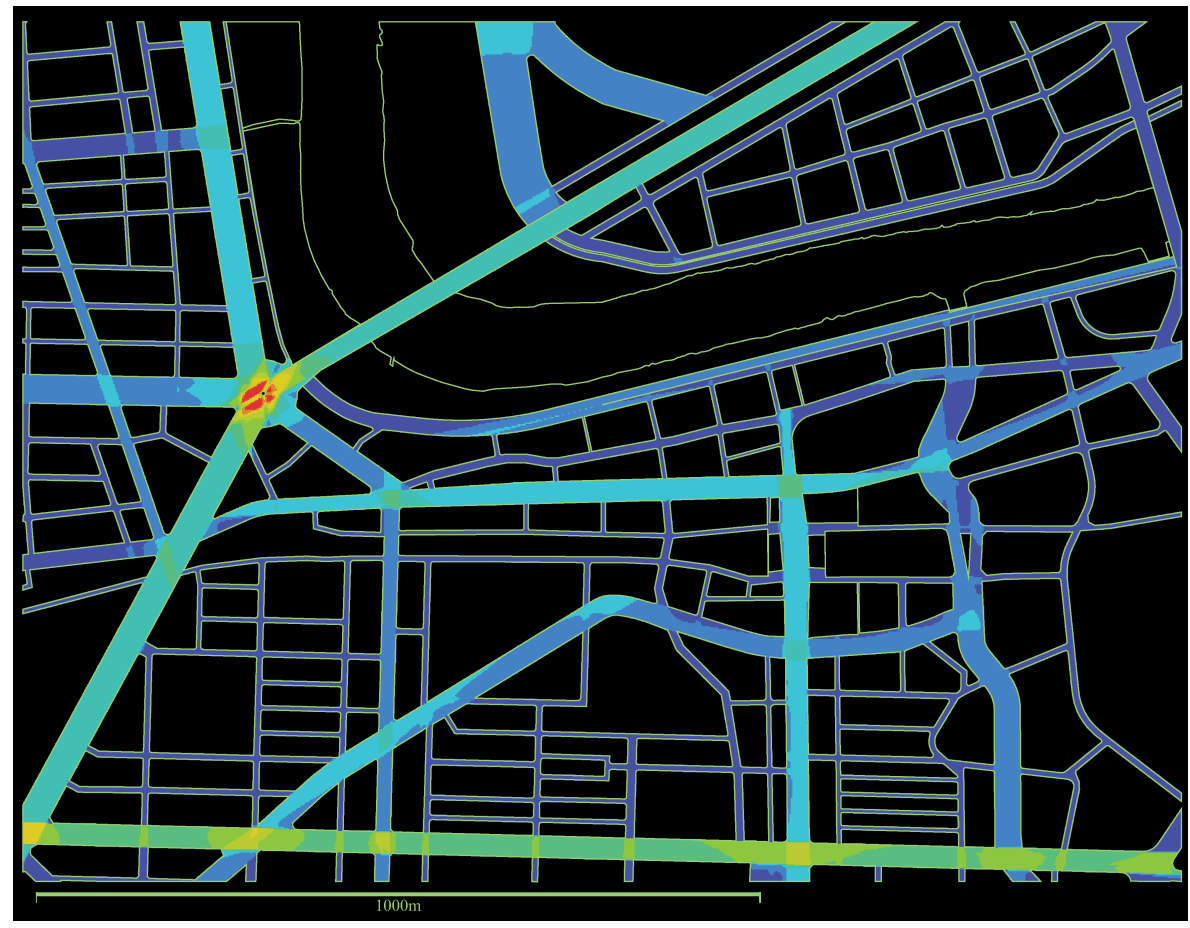




\section{Discusion}

\section{Local Levels of Spatial Integration (In-Situ Visualization)}

Visiting the site redefined entirely a new attitude towards the night markets' survey. The in-situ observation practice intended to establish a visual contrast of the obtained sketch from the computarized simulation with the real condition. In this part of the research was necessary to put into practice a method of evaluation for this the urban form. The interrogations were: What does this city's form actually mean for the people who make their living there? What findings we expect to obtain that can contribute to the upgrade of a human-centered smart city approach? Is it possible to find a potential hidden value to guide the criterias to strenghten the service design theory of this commercial zones?

During the experiment was practiced, the research intention was to describe from visual perception the correspondent pedestrian flow visualization on the simulation images based on an objective criteria. Thus, the discussion of this report mainly addresses the identified similarity between the spatial characteristic of the night market's streets and the outward form linked with the obtained result from the simulation diagrams. The main criteria utilized was the value calculated in depthmapX versus the amount of pedestrians reported by the in-situ observation. It does not narrate the individual image of the group of stores around the highlighted sections but intends to demonstrate the need of user-oriented design response for the current situtation possible issue.

In short, here are presented the discussions for every spatial integration condition and its relationship with the in-situ pedestrian concentration. It also presents the geographic point for every scenario in a simplified map for better understanding of the location into the knotty street pattern. Then, as product of this discussion it can be noticed a specific construction in the spatial arrangements as a major piece of commercial architecture.

Highest Level:
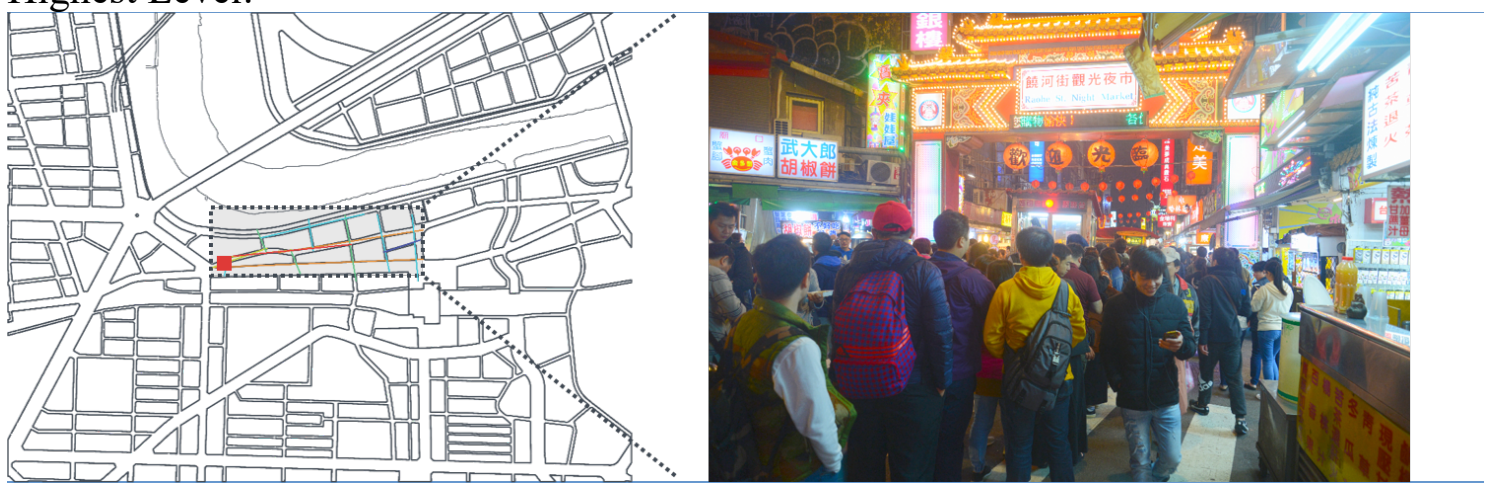

Raohe Night Market posseses very well identified entrances. This facilitates a handy spatial orientation for new visitors, but on the other hand it saturates the two known entryways causing the crowd to squeeze when entering into the night market's main strip. At every instant, the experience for the highest level of integration value might represent the lowest level of comfortability for visitors, long queues are seen and there is no clear separation boundaries among the large variety of products being sold. 

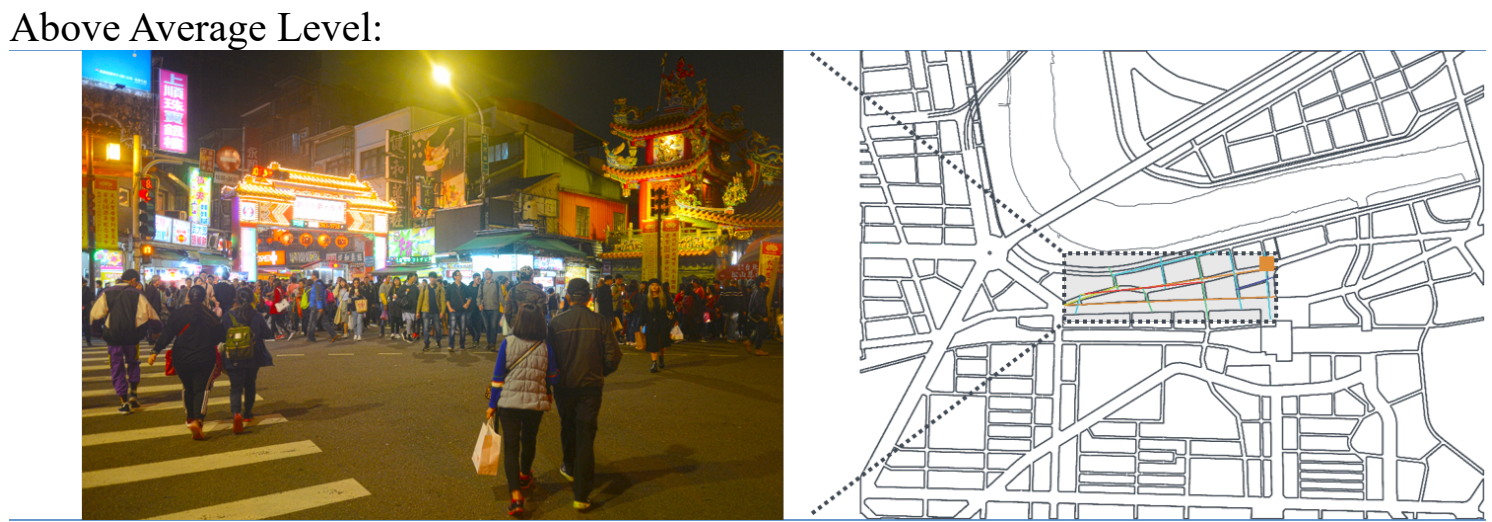

Few meters away from the focus points; where people concentrate to access the night market or nearby important reference buildings such as temples and popular stores; the integration value fades to "above average", which still represents high concentration of individuals in narrow square meters; but beyond the limits of the surrounded area, it starts to scatter towards other focus points such as public transportation hubs: bus, train or the entrance of the subway station. Such concentration of pedestrians is subject to constant changes based on timing, presence of ambulant vendors and weather condition.

Average Level:
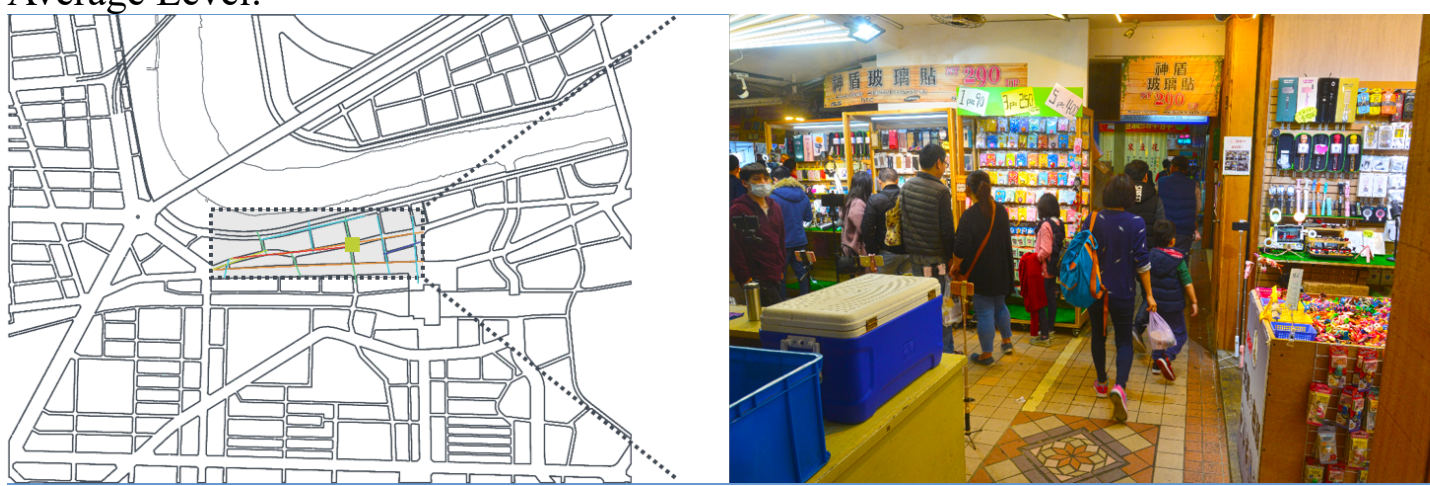

Although there's an implicit clarity or legibility to this level of integrity, this feature is quite dificult to find on a regular street pattern. By no means we must assume that the only important property of stands concentration on the street, is the dynamics of the multiple services offered. All mixed with half exposed halls from the buildings we also can observe multiple forms of commercial businesses, also a good variety of products and services. They are all possible thanks to the traditional "arcade" that wraps up multiple businesses protecting them from external factors such as bad weather.

Many kinds of cues are used to distinguish the implicit boundaries in this level of integrity: the visual sensations of color, shape of the signs, motion of the ambulant vendors, or polarization of light, as well as other senses such as smell, sound, touch, kinesthesia, sense of location and way-finding. All of them together provide a rich insight of the dynamics of what is considered the core of this retail establishments.

The spaces comprenhending this structures are: the "traditional Taiwanese arcade", the interior spaces of some big scale retail stores and other long lasting businesses. 

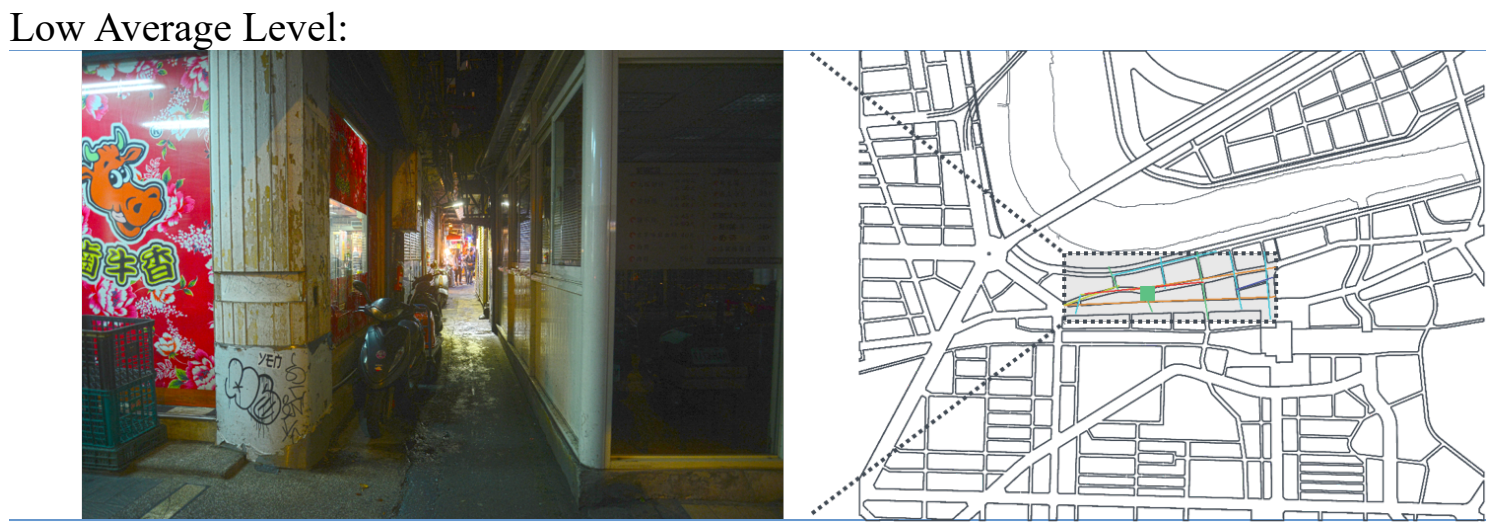

Under the average level of integrity are the lanes and alleys which are more active during daytime, these paths loose vitality during night time and they are rarely visited when other areas of the night market still are vivid, lively and bustling with noise.

Low Level:
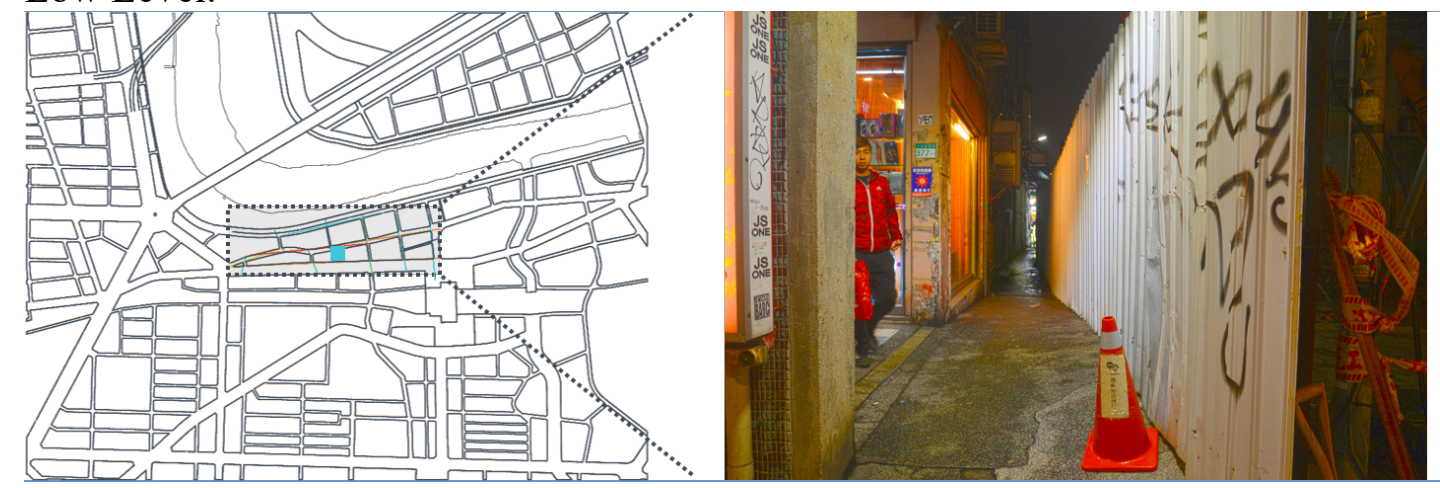

These spaces are the "leftovers" unfinished construction sites or other empty properties, they are not under any kind of official use but their characteristic implies this narrow allies might serve as emergency exits and mitigation routes in case of disasters.

\section{Lowest Level:}
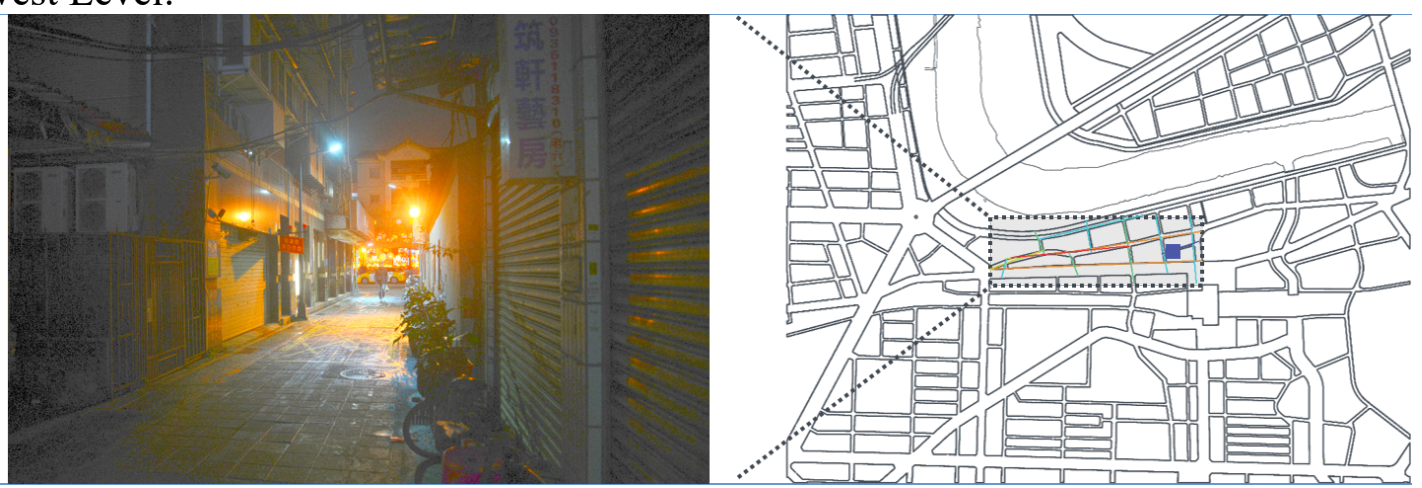

Frequently abandoned and exempted from a more public access are these lanes among the most evaded areas in the night market, most of them are the rear backdoor that link any service exit to other emergency alleys. Unfortunately, these exits are nearby very dirty drainage or exposed sewer. 


\section{Conclusions}

The presented investigation is the most recent product of an ongoing research carried out by the laboratory of service design and smart interactive technologies in Taipei Tech. It pursues to integrate the human-centered design discipline with spatial computing technologies in a new integral way of addressing computing disciplines related to space. Space Syntax is indeed part of the domains of spatial computing simulation. This research suggests an extended application of user's observation and its relationship with space syntax analysis.

Developing widespread real-time night market's data based on human-centered smart technologies optimizes private and public assets by instantly improving urban operations and contributing to an advanced strategy for smart city environment approach. This has led to numerous breakthroughs in urban government models, innovative service models, and commercial development. Participants involved are capable to intelligently detect more events and reduce average handling time avoiding ambiguous landmarks, node points and paths that mislead the visitor. This research effort focuses on including other resources and innovative methods and tools into pedestrian navigation services in order to improve visitors' spatial routing information. The most important finding obtained in this research is the comparison of the selected area in-situ observation with the analysis result to verify the implementation of the computing outcome which supports the idea of visualizing visitors' spatial experience.

Within the main findings obtained by this research it can be emphasized the role of integration as a key factor in the pedestrian movement across night markets' street network and therefore, these measures could aid to identify the streets with low accessibility levels. It is conceivable to adequately anticipate the concentration of individual pedestrian flow and to adequate the spaces developments. At the same time, considering spaces movement and shoprunning, the reasonable human stream appropriation in the built-environment shopping spaces will improve the business homogenization.

Specific targeted conclusions for the objectives presented in this research are as following:

1. Visitor's Spatial Experience Attributes: the role of integration as a key factor in the pedestrian movement across night markets' street network and therefore, these measures could aid to identify the streets with low accessibility levels.

2. Human-Centered Design: it is conceivable to anticipate the concentration of individual pedestrian flow and to adequate the spaces needs. At the same time, considering spaces movement and shop-visiting behavior.

3. "Servicescape" Visualization: the visitor's wandering flow during shopping proved to be a useful data source to improve the shops' visibility homogenization. It also serves as an innovative tool to propose an adequate "servicescape" based on the space syntax analysis.

4. Smart City: Emphasizes the approach of how this research has successfully shown an innovative source of data interpretation to visualize the user's behavior in space. Knowing this vital information, it can provide an abundant feedback to future smart city planning and development from a human-centered design perspective. 


\section{Future Works and Suggestions}

Nowadays, due to the rapidly evolving technological advances; the smart city approach has been mostly addressed from eminently the automation technologies perspective and falling short into implementations, leaving an important gap with the users' empathy and sensorial experiences. Further application of both methods included as design tool can directly improve the understanding of pedestrian's movement levels through different spaces. It can play an important role from other perspective to contribute in the smart city theory strengthening supported by a user-centered design perspective. For now, we have exclusively worked with a small scale site to test the implementation of the recommended practical procedure. Then, as suggestion obtained for future works our team highly advises to prepare enormous computing power to analyze larger streets' networks, including their spatial characteristics for major cities regions where this research can provide a better and more integral smart city approach for future urban planning.

\section{References}

[1] Era, R. T. (2012). Improving pedestrian accessibility to public space through space syntax analysis. Paper presented at the Proceedings: Eighth international Space Syntax Symposium Santiago, PUC.

[2] Golledge, R. G. (1997). Spatial behavior: A geographic perspective: Guilford Press.

[3] Hillier, B. (2007). Space is the machine: a configurational theory of architecture: Space Syntax.

[4] Hillier, B., Leaman, A., Stansall, P., Bedford, M. J. E., Planning, p. B., \& design. (1976). Space syntax. 3(2), 147-185.

[5] Hillier, B., Penn, A., Hanson, J., Grajewski, T., Xu, J. J. E., planning, P. B., \& design. (1993). Natural movement: or, configuration and attraction in urban pedestrian movement. 20(1), 29-66.

[6] Li, Y., Xiao, L., Ye, Y., Xu, W., \& Law, A. J. T. M. (2016). Understanding tourist space at a historic site through space syntax analysis: The case of Gulangyu, China. 52, 30-43.

[7] Mansouri, M., \& Ujang, N. (2017). Space syntax analysis of tourists' movement patterns in the historical district of Kuala Lumpur, Malaysia. Journal of Urbanism: International Research on Placemaking and Urban Sustainability, 10(2), 163-180.

[8] Markopoulos, P., Read, J. C., MacFarlane, S., \& Hoysniemi, J. (2008). Evaluating children's interactive products: principles and practices for interaction designers: Elsevier.

[9] Millonig, A., \& Schechtner, K. (2007). Developing Landmark-Based PedestrianNavigation Systems. IEEE Transactions on Intelligent Transportation Systems, 8(1), 43-49. doi:10.1109/TITS.2006.889439

[10] Özbil, A., \& Peponis, J. J. A. (2007). Modeling street connectivity and pedestrian movement according to standard GIS street network representations. 18, 02.

[11] Turner, A., Penn, A., Hillier, B. J. E., planning, P. B., \& design. (2005). An algorithmic definition of the axial map. 32(3), 425-444.

[12] Xiana, H. J. P. E. (2017). Simulation of Pedestrian Flow in Traditional Commercial Streets Based on Space Syntax. 205, 1344-1349. 